\title{
Los retos de investigar en la universidad
}

\author{
Autor: José Jesús Guerrero Rojas \\ Universidad César Vallejo, UCV \\ iguerrero28@ucvvirtual.edu.pe \\ Lima, Perú \\ https://orcid.org/0000-0002-2346-5797
}

\section{Resumen}

La investigación es uno de los objetivos principales que persigue la universidad, siendo esta última el espacio donde, aplicando métodos y estrategias investigativas, se podrán dar y plantear soluciones a los problemas que afectan a la sociedad, como sostienen Aguilar, Aréstegui y Vergara (2019); Arellano-Sacramento, Hermoza-Moquillaza, Elías-Podestá y Ramírez-Julca (2018); y Sánchez (2016). El propósito del presente ensayo es ofrecer respuestas a los conflictos que se presentan en la población universitaria para investigar, conocer porque, por ejemplo, los estudiantes temen investigar, más allá de los trabajos monográficos o de grado, según lo afirma Ishiyama (2018). Esta investigación concluye que, entre las principales razones, destacan la gran variedad y complejos términos empleados en los cursos de "metodología de la investigación" a los que se debe agregar la poca formación e interés de los docentes que dictan estas asignaturas. superior.

Palabras clave: investigación científica; universidad; reto; educación

Código de clasificación internacional: 5802.07 - Formación profesional.

Cómo citar este ensayo:

Guerrero, J. (2021). Los retos de investigar en la universidad. Revista Scientific, 6(21), 282-300, e-ISSN: 2542-2987. Recuperado de: https://doi.org/10.29394/Scientific.issn.2542$\underline{\underline{2987.2021 .6 .21 .15 .282-300 ~}}$

Fecha de Recepción: 15-02-2021
Fecha de Aceptación: 26-06-2021
Fecha de Publicación: 05-08-2021 


\title{
The challenges of researching at the university
}

\begin{abstract}
Research is one of the main objectives pursued by the university, the latter being the space where, by applying investigative methods and strategies, solutions to problems that affect society can be given and proposed, as argued by Aguilar, Aréstegui and Vergara (2019); Arellano-Sacramento, HermozaMoquillaza, Elías-Podestá and Ramírez-Julca (2018); and Sánchez (2016). The purpose of this essay is to offer answers to the conflicts that arise in the university population to investigate, to know why, for example, students fear to investigate, beyond monographic or degree works, according to Ishiyama (2018). This research concludes that, among the main reasons, stand out the great variety and complex terms used in the courses of "research methodology" to which must be added the little training and interest of the teachers who teach these subjects.
\end{abstract} education.

Keywords: scientific investigation; university; challenge; higher International classification code: 5802.07 - Vocational training.

Date Received: 15-02-2021
Date Acceptance: 26-06-2021
Date Publication: 05-08-2021 


\section{Introducción}

¿Por qué existe poco o nulo interés por investigar dentro del contexto universitario?. Esta suele ser una pregunta recurrente cuando se trata de entender las razones por las cuales estudiantes y docentes universitarios, ya sea en la etapa formativa como al egresar, manifiestan un marcado desinterés por investigar y generar conocimiento.

Siendo uno de los principales objetivos de la universidad peruana la generación de conocimiento mediante el desarrollo y ejecución de diversos proyectos de investigación científica, expuesto en el Congreso de la República (2014a): en la Ley Universitaria; y la Superintendencia Nacional de Educación Superior Universitaria (SUNEDU, 2018): en el Perú este proceso sigue siendo insuficiente para permitir su posicionamiento dentro de los estándares internacionales. Se debe señalar que en los últimos años se evidencia un considerable incremento en la producción científica universitaria según Stefos (2019); y SUNEDU (2020): lo que demuestra el compromiso de las universidades peruanas para ceñirse a lo establecido por la Ley Universitaria N. ${ }^{\circ} 30220$.

Entendidas para Purizaca-Rosillo, Cardoza-Jiménez y Herrera-Añazco (2016a): como "medio para generar conocimientos útiles para el desarrollo de las naciones" (pág. 73); en centros de producción de conocimiento, las universidades son las responsables de la formación de profesionales con la capacidad de dar soluciones a los distintos problemas de la sociedad. En este sentido, Aguilar, Aréstegui y Vergara (2019a): añaden que también son "instituciones, en donde la investigación y la docencia, deben conformar una unidad de acción para procurar que el estudiante interactúe con su propia realidad y la realidad nacional" (pág. 45). Es necesario tener en cuenta que la educación superior universitaria no solo consiste en recibir y transmitir conocimientos sino de generarlos, buscando soluciones a los distintos problemas que presenta la sociedad. 
En un marco más amplio, podemos decir que la investigación, especialmente la relacionada con la ciencia, es un proceso cuya principal finalidad es conseguir conocimientos que permitan comprender y entender la realidad, además de solucionar problemas diversos (científicos, sociales, empíricos o filosóficos entre otros) y que se desarrolla mediante la aplicación del método científico, definido como un conjunto de pasos que incluyen técnicas de observación, razonamiento y generación de posibles respuestas, experimentación programada, medios eficientes, difundir y comunicar los resultados obtenidos.

En la actualidad, la calidad del servicio educativo ofrecido por el sistema universitario peruano se mide, entre otros requisitos, por el nivel y volumen de trabajos de investigación realizados en la etapa formativa tanto por los estudiantes como por los docentes, a lo que afirma Rodríguez (2013): hay que añadir la necesidad y exigencia de publicar artículos científicos en revistas indizadas o indexadas por parte de las instituciones universitarias. Bajo este criterio, el autor analiza la problemática de la realidad universitaria desde la evidente ausencia de cultura de investigación en los estudiantes universitarios, sobre todo la formativa. Asimismo, realiza una comparación entre la docencia y la investigación como parte fundamental en el proceso investigativo y de generación de conocimientos dentro del ámbito universitario.

Mencionando a Ishiyama-Cervantes (2017a): en el Perú la investigación científica se presenta como "[...] actividad ardua y complicada" (pág. 115); en la que los conocimientos complicados e innecesarios convierten a la actividad en algo temible y que cause fobia entre los interesados. Prueba de ello, fue la investigación realizada por Yace, Guerrero y Delgado (2017): quienes reportan la indiferencia de estudiantes de la escuela profesional del área de salud en su actitud para la realización de trabajos de investigación relacionados con su especialidad.

Los investigadores también señalan que esta es una constante que se 
repite en otras instituciones de educación superior universitaria. En relación, Arellano-Sacramento, Hermoza-Moquillaza, Elías-Podestá y Ramírez-Julca, (2018): encontraron que la actitud de los estudiantes fue regular.

¿Por qué la indiferencia?; ¿Por qué la investigación es considerada algo tedioso y complicado?; ¿De quién depende, estudiantes o docentes?. Estos y otros más pueden ser considerados como los factores que impiden la existencia de una verdadera cultura de investigación dentro del ámbito de la educación superior peruano. La experiencia personal, tanto en el pregrado como en posgrado, nos muestra que existe un marcado rechazo por parte de los estudiantes a investigaciones, aun sabiendo y siendo conscientes de que de ello depende la obtención de su grado académico o su título profesional. Ante este panorama, con algunas excepciones en el ámbito de las ciencias básicas y exactas, es poco probable que los egresados continúen investigando luego de abandonar las aulas.

El presente ensayo tuvo como propósito, conocer en primera instancia, los retos y desafíos que enfrenta el estudiante universitario al momento de realizar una investigación y, a partir de ello, proponer algunas respuestas que permitan explicar el aparente desinterés por investigar y generar conocimiento.

\section{Desarrollo}

\subsection{Ley $N^{\circ} 30220$ - Nueva Ley Universitaria}

Promulgada en julio del 2014, la nueva Ley Universitaria, N. ${ }^{\circ} 30220$, supuso un cambio radical en las exigencias que permiten el funcionamiento de las universidades peruanas, así como en la calidad que deben ofrecer estas instituciones a la población estudiantil del país. Entre algunos de sus articulados, la norma estableció la creación de la Superintendencia Nacional de Educación Superior Universitaria (SUNEDU), en reemplazo de la Asamblea Nacional de Rectores (ANR). Las principales funciones de este nuevo organismo fue la de evaluar a cada universidad peruana para otorgar el 
Instituto Internacional de Investigación y Desarrollo Tecnológico Educativo INDTEC, C.A.

DOI: https://doi.org/10.29394/Scientific.issn.2542-2987.2021.6.21.15.282-300

OAI-PMH: http://www.indteca.com/ojs/index.php/Revista Scientific/oai

Ensayo Original / Original Essay

correspondiente licenciamiento institucional. La actividad investigadora, demostrada en la formulación y ejecución de diversos proyectos de investigación con su correspondiente publicación en revistas especializadas como exigencia requerida.

Estas exigencias pretenden mejorar la calidad de la educación universitaria en nuestro país y cumplir con una de sus principales funciones: investigar y, por ende, generar conocimiento, sin dejar de lado la formación académica y profesional de los jóvenes que asisten a sus aulas.

Ya desde la definición, la nueva Ley Universitaria, se precisa el rol de la universidad en materia de investigación científica (Definición de universidad; Fines de la universidad; y Funciones de la universidad). Pero la obligatoriedad de realizar esta actividad se establece de manera precisa en el Capítulo VI, de esta Ley, según Congreso de la República (2014b):

La investigación constituye una función esencial y obligatoria de la universidad, que la fomenta y realiza, respondiendo a través de la producción de conocimiento y desarrollo de tecnologías a las necesidades de la sociedad, con especial énfasis en la realidad nacional. Los docentes, estudiantes y graduados participan en la actividad investigadora en su propia institución o en redes de investigación nacional o internacional, creadas por las instituciones universitarias públicas o privadas (art. 48).

En referencia al tema, Salmi (2009); y la Comisión Europea (2003), citados por Superintendencia Nacional de Educación Superior Universitaria (SUNEDU, 2018a): indican que "la participación de las universidades en la producción de conocimiento a través de la investigación contribuye eficazmente al desarrollo de un sistema nacional de innovación, abierto a la creación, aplicación y difusión de nuevas tecnologías" (pág. 23); la participación de la universidad en la generación de información confiable, en todas las áreas del conocimiento, es de vital importancia, pues en ellos radica la neutralidad y objetividad, libre de apasionamientos e intereses creados, que 


\section{Ensayo Original / Original Essay}

todo proceso científico requiere.

En esa misma línea, Gago (2016), citado por SUNEDU (2018b): menciona que "las universidades y los centros de investigación son los encargados de realizar la investigación básica de un país y contribuir a formar científicos de alto nivel" (pág. 23); como vemos, la participación de la universidad en la generación de información confiable en todas las áreas del conocimiento es de vital importancia pues en ellos radica la neutralidad y objetividad, libre de apasionamientos e intereses creados que todo proceso científico requiere

La necesidad de realizar algunas mejoras a la Ley, son realizadas por Valles (2015): quién reflexiona sobre "la garantía del futuro éxito profesional de los jóvenes estudiantes depende, en gran parte, de la calidad humana y profesional de sus profesores" (pág. 79); precisando el impacto y futuro de mejorar la calidad educativa que se da en las distintas casas de estudio.

Medir la calidad y producción científica de las universidades implica evaluar la cantidad y calidad de artículos científicos publicados en revistas especializadas y reconocidas a nivel mundial, calificadas bajo estándares internacionales. Aquí, debe diferenciarse la tendencia que solo aquellas investigaciones $\mathrm{o}$ artículos publicados en grupos editoriales grandes $\mathrm{O}$ repositorios como Scopus y otros, sirven de punto de partida para considerar que un artículo es de calidad, más aún si se tiene en cuenta que publicar en este tipo de revistas demanda una inversión económica muy onerosa.

Sin embargo, la relación entre investigación y publicación, y la calidad de esta, es necesaria para reconocer un verdadero aporte de la universidad al conocimiento científico. El trabajo de Bendezú, Hurtado, Medina y Aguilar (2015): analiza y evalúa la relación entre la capacitación en investigación y la publicación de artículos científicos de los estudiantes universitarios, específicamente en el área de ciencias biomédicas, considerando que la investigación es un componente esencial en la formación médica para el 


\section{Ensayo Original / Original Essay}

avance científico y el cuidado de la salud.

Igualmente, Gonzales-Saldaña, Chavez-Uceda, Lemus-Arteaga, SilvaOcas, Galvez-Olortegui y Galvez-Olortegui (2018): al realizar un análisis bibliométrico de las publicaciones presentadas por los docentes de la Universidad Nacional de Trujillo, concluyen que es "necesaria la implementación de estrategias que fomenten la producción científica involucrando a estudiantes, docentes, autoridades y egresados, así como la instauración de redes de colaboración que complementen las estrategias implementadas por CONCYTEC" (págs. 128-129).

Las deficiencias de nuestro sistema universitario en materia de generación de conocimiento y publicaciones se reflejan en nuestra presencia en los distintos rankings latinoamericanos o mundiales. Las universidades peruanas están ubicadas en lugares bastante alejados, no llegan a colocarse siquiera entre los 100 primeros y lograr avanzar de manera significativa en el mediano paso parece imposible.

En este panorama rescatamos lo precisado por Sánchez (2016a): quién señala que "el desafío más importante para la universidad peruana es lograr que se cumpla con los estándares mínimos exigidos le que permitan estar ubicada en un lugar decoroso en los rankings mundiales sobre investigación universitaria". Menuda labor la que espera a todos los involucrados en investigar en nuestro país, pues implica una cuota mayor de esfuerzo por mejorar la calidad de los proyectos de investigación y sobre todo de los artículos científicos a ser publicados en revistas de prestigio.

\subsection{Retos}

Son múltiples los retos a considerar cuando se habla de investigación científica. Son numerosos los factores que influyen en el desarrollo de un compromiso real con la investigación científica que para Aguilar, Aréstegui y Vergara (2019b); Ishiyama-Cervantes (2017b); y Sánchez (2016b): son el 
Financiamiento; capital humano; ausencia de la cultura de investigación; limitado nivel de capacitación y perfeccionamiento de los docentes; imprecisa selección del tipo y método de investigación, falta de guía y sesgos en los temas a investigar y que contribuyan al desarrollo científico, tecnológico, y que tengan un impacto en la sociedad, expuesto en la tabla 1.

Tabla 1. Factores que influyen en la investigación científica universitaria.

\begin{tabular}{|l|}
\hline \multicolumn{1}{|c|}{ Factores } \\
\hline Financiamiento. \\
\hline Falta de una verdadera cultura de investigación. \\
\hline Docentes poco preparados en investigación o que no investigan. \\
\hline Desconocimiento de cómo formar investigadores y docentes desde el currículo. \\
\hline Falta de motivación para realizar trabajos de investigación. \\
\hline Poca apertura, por parte de los docentes, hacia los requerimientos de los estudiantes. \\
\hline Desconocimiento en la selección método de investigación. \\
\hline Obligación por investigar. \\
\hline
\end{tabular}

Fuente: Aguilar, Aréstegui y Vergara (2019); Ishiyama-Cervantes (2017); Sánchez (2016).

Hacia finales del año 2019, Bajak (2019): realizó un informe sobre la realidad y potencial de la investigación científica en nuestro país. Luego de entrevistar a algunos investigadores el autor indica que una de las dificultades radica en los aspectos burocráticos, a la carencia de apoyo gubernamental y del obsoleto sistema educativo peruano.

Respecto del apoyo gubernamental, el informe hace mención de que la inversión en investigación científica por parte el gobierno es muy poca, donde un pequeño porcentaje $0,12 \%$ del Producto Bruto Interno $(\mathrm{PBI})$ se invierte en ciencia y tecnología comparado con el $0,36 \%$ en Chile, el $1,27 \%$ en Brasil y el 2,8\% en los Estados Unidos.

\subsection{Recurso humano: estudiantes y docentes}

Otro factor fundamental es el recurso humano y su debida preparación o capacitación para investigar, donde los cursos relacionados son dictados de 
manera confusa y que para Ishiyama (2018a):

Formar investigadores no son necesarios cursos complejos, la sencillez es la mejor forma. Las acciones complicadas con definiciones innecesarias causan fobia entre los interesados en iniciarse en esta tarea. Los cursos sencillos con más práctica que teoría dan buenos resultados (pág. 34).

Es preciso indicar que desde su nacimiento, el ser humano investiga, analiza y evalúa su entorno, reconoce su cuerpo, toca, palpa, saborea (usa su boca como principal reconocedor de su medio circundante), dirigiéndose siempre hacia aquello que le llama la atención. Interpretando a Ishiyama (2018b): a medida que va creciendo, razona, evalúa, aplica el error y ensayo utilizando esta investigación empírica para incrementar y seleccionar la adquisición de conocimientos.

En el espacio de la educación superior, cabe preguntarse si los estudiantes ¿investigan por obligación, para cumplir con un requisito académico, o lo hace porque le gusta?. Generalmente, la respuesta es que todos los trabajos de investigación se realizan por obligación, con el propósito de cumplir con el requisito establecido por algún curso o materia y obtener la calificación aprobatoria respectiva. No existe cultura de investigación formativa. La gran mayoría de estudiantes no tiene idea de lo que está investigando, se realizan "a la carrera" y como es usual "a la ultima hora". Llegado el momento de realizar las tesis de grado o titulación el fenómeno se repite, se hace por obligación, y al graduarse, la investigación queda relegada y hasta olvidada, salvo excepciones.

Otro reto por considerar es el desconocimiento por parte del alumno sobre ¿qué investigar?. Al no haber tenido un proceso formativo en investigación, los estudiantes no tienen ni idea de hacia dónde dirigir sus intereses científicos y navegan en la incertidumbre y la desesperación de no saber "que investigar". La búsqueda del tema es quizás el principal obstáculo 
para vencer, siempre quieren tener una investigación que no demande mucho tiempo de ejecución, que no cueste mucho, aunque ello signifique investigar sobre algo que no es de su interés, con lo que no hay un compromiso real.

Todo ello, se agrava cuando entre los requisitos o consideraciones para elaborar la investigación de grado se incluye el requerimiento de que estas deben ser "novedosas e innovadoras" con lo que se incrementan sus miedos, temores y su rechazo a generar conocimientos.

Desde los primeros ciclos, los estudiantes deben ser "enfrentados" al mundo de la investigación científica, donde aprendan a buscar información bibliográfica adecuada -a pesar de las facilidades tecnológicas existentes, los estudiantes no saben cómo buscar información relevante- y relacionada con un tema de interés. Debe comenzar con la elaboración que articule la monografía y progresivamente ir escalando hasta el momento de elaborar y ejecutar su proyecto de investigación y finalmente redactar la tesis y uno -o varios- artículos científicos. Aspecto fundamental es la constante motivación que reciban.

Y ¿el docente?; ¿Cuál es su rol?. Definitivamente, este es el componente que tiene mayor responsabilidad dentro del proceso investigativo. Corresponde a los docentes incentivar y motivar, además de ser guías, a los estudiantes en la creación de una cultura de investigación. La realidad nos indica que muchos de ellos no están debidamente capacitados para investigar, son reacios a elaborar y ejecutar proyectos que generen conocimientos con lo que el estudiante, y la institución, se ve perjudicado. Además, muchos tienen ciertos parámetros fijos y no muestran la apertura necesaria para brindar todas las alternativas a sus estudiantes.

Para Díaz (2019): ser investigador es tarea elemental de "[...] todo docente porque de él depende el incentivo que pueda ofrecer a los estudiantes para lograr aprendizajes óptimos en pro de la investigación y, por ende, en la formación de competencias investigativas" (pág. 331). El docente debe ser la 
primera puesta o ventana a la que el estudiante pueda acceder para satisfacer su inicial curiosidad científica. Además, es quién, de manera empática y amigable, se convierta en el guía y mentor que dirija en forma adecuada los intereses científicos de sus estudiantes teniendo un a mente abierta para resolver cualquier inquietud estudiantil.

Para Ishiyama-Cervantes (2017c): también se debe considerar lo tedioso que resultan los denominados cursos de "metodología de la investigación" (pág. 116); que se traducen en la presentación de conceptos obsoletas y muy técnicas, sin aplicación útil y práctica desmotivando a los estudiantes, que empiezan a considerar a la investigación como algo complicado En relación con los métodos de investigación, Sánchez (2016c): indica que "[...] la imprecisión o escasa comprensión de un método de investigación puede afectar los diseños y por tanto la validez, los alcances y los resultados de una investigación" (pág. 4). En esta línea, para Rojas (2015): es variada y confusa la lista de términos complejos.

En este ámbito, Reynosa, Serrano, Ortega-Parra, Navarro, CruzMontero y Salazar (2020): recomiendan que para la formación de investigadores es necesario contar con educadores o docentes con un alto dominio didáctico, capaces de emplear las estrategias y recursos pedagógicos para que los estudiantes y futuros investigadores adopten la cultura de investigación acorde con las necesidades socioculturales de su entorno.

Las deficiencias en el sistema educativo universitario se reflejan en los estudios de posgrado cuando los estudiantes de maestría, por ejemplo, encuentran muchos obstáculos y trabas para elaborar y diseñar su proyecto de investigación, pues se mueve en la disyuntiva de ejecutar un modelo cuantitativo o cualitativo, cada una con sus características, complejidades y requerimientos propios haciendo que el estudiante rechace cualquier actividad investigativa por lo tedioso de la misma. 


\subsection{Financiamiento}

Como ya se ha señalado, el financiamiento o la obtención de recursos económicos financieros para investigar es uno de los principales obstáculos que deben enfrentar los investigadores peruanos. En el sector público, los presupuestos brindados por el Estado están muy lejos de cumplir con los requerimientos para la investigación, por lo que un alto porcentaje de proyectos terminan siendo financiados por recursos externos, obtenidos por gestiones propias de los investigadores, quienes como se puede deducir también deben disponer de sus tiempos para también financiar sus investigaciones. Al respecto, Aguilar, Aréstegui y Vergara (2019c), indican que:

[...] Son las universidades públicas las que tienen que buscar su propio financiamiento para que puedan cubrir todos los gastos que les genera poder realizar una investigación y así seguir manteniéndose en el ranking de las mejores universidades que realizan investigaciones para mantenerse a la vanguardia (pág. 46).

En este mismo orden de ideas, Purizaca-Rosillo, Cardoza-Jiménez y Herrera-Añazco (2016b): señalan que la producción científica depende los recursos financieros pues posibilita la realización de investigaciones básicas y complejas además de permitir la publicación en revistas de mayor impacto, sobre todo aquellas en las que se requiere pago por publicación.

De otro lado, las estadísticas muestran al Perú como una de las naciones que menos recursos destina a la educación y a la investigación científica. Cambiar esta realidad requiere que se den las facilidades y recursos necesarios para que las universidades puedan disponer de autonomía económica para ejecutar distintos proyectos.

Un aspecto a considerar, es el relacionado con la gestión y administración de los recursos otorgados a las universidades para investigar. El canon minero es un ejemplo de ello, pues a pesar de los importantes montos que se dan, estos no son debidamente utilizados, pues no existe la 
capacitación pertinente para la elaboración de proyectos ejecutables o la cantidad suficiente de estos que reporten resultados satisfactorios.

\section{Conclusiones}

Los estudiantes ya ni sienten o muestran interés para adquirir -y generar- conocimientos que sustenten su formación profesional; han dejado de lado la búsqueda bibliográfica y la lectura. La existencia del mundo globalizado e Internet les ha facilitado -en cierta medida- el encontrar la información requerida para sus propósitos; a ello hay que añadirle la incultura del "copiar y pegar". Remediar esta situación implica un trabajo de estimulación y cuestionamiento constante por parte del docente tratando de incentivar en el alumno la necesidad de conocer más. Ello puede ser desarrollado preguntando y confrontando las apreciaciones dadas en clases, así como dejando interrogantes "en el aire" a las que el estudiante debe encontrar respuesta y/o solución.

La motivación, es un factor importante en el desarrollo y formación de los futuros investigadores. Esta gran mayoría de estudiantes no encuentra el interés necesario por parte de sus docentes en la búsqueda de respuestas a sus inquietudes y potencialidades de investigación. El docente debe estar dispuesto a resolver o al menos atender a las distintas inquietudes surgidas por los estudiantes en su proceso de formación investigativa, motivándolos a seguir indagando y buscar las respuestas que su investigación requiera.

La falta de perseverancia de los alumnos es otro gran problema. Los futuros investigadores se rinden ante el primer obstáculo, considerado como un fracaso, desalentándolos a seguir en el intento. En este aspecto papel importante lo juega el docente quién debe reconocer y desarrollar la potencialidad de investigador del alumno, incentivándolos a continuar por la senda del cuestionamiento constante. Una forma de potenciar estas habilidades puede ser brindándoles a los alumnos información sobre su tema 
de interés, incluyendo datos sobre referencias bibliográficas, para que ellos busquen en bibliotecas; o proporcionándoles el o los artículos directamente.

\section{Referencias}

Aguilar, B., Aréstegui, C., \& Vergara, C. (2019a,b,c). Diagnóstico de la investigación universitaria en el Perú. Journal of the Academy, (1), 44-56, e-ISSN: 2707-0301. Recuperado de:

https://doi.org/10.47058/joa1.5

Arellano-Sacramento, C., Hermoza-Moquillaza, R., Elías-Podestá, M., \& Ramírez-Julca, M. (2018). Actitud hacia la investigación en la facultad de ciencias de la salud de la Universidad Privada Norbert Wiener, 2017. Revista de Investigación de la Universidad Norbert Wiener, (7), 47-58, e-ISSN: 2663-7677. Recuperado de: https://revistadeinvestigacion.uwiener.edu.pe/ojs/index.php/revistauwie ner/article/view/6

Bajak, A. (2019). What should Peru do to improve its science?. Nature, 576, S65-S67, e-ISSN: 1476-4687. Recuperado de: https://doi.org/10.1038/d41586-019-03844-2

Bendezú, G., Hurtado, S., Medina, C., \& Aguilar, P. (2015). Apreciación sobre capacitación en investigación y publicación científica en estudiantes universitarios. Investigación en Educación Médica, 4(13), 50-51, e-ISSN: 2007-865X. Recuperado de:

https://www.redalyc.org/articulo.oa?id=349736307009

Congreso de la República del Perú (2014a,b). Ley Universitaria nro. 30220.

Lima, Perú: Departamento de Investigación y Documentación Parlamentaria del Congreso de la República.

Díaz, Y. (2019). Práctica en las Líneas de Investigación y su Importancia para la Educación Universitaria. Revista Scientific, 4(14), 328-346, eISSN: 2542-2987. Recuperado de: 
https://doi.org/10.29394/Scientific.issn.2542-2987.2019.4.14.16.328$\underline{346}$

Gonzales-Saldaña, J., Chavez-Uceda, T., Lemus-Arteaga, K., Silva-Ocas, I., Galvez-Olortegui, T., \& Galvez-Olortegui, J. (2018). Producción científica de la facultad de medicina de una universidad peruana en SCOPUS y Pubmed. Educación Médica, 19(supp 2), 128-134, eISSN: 1575-1813. Recuperado de:

https://doi.org/10.1016/j.edumed.2017.01.010

Ishiyama, R. (2018a,b). La equivocada creencia que iniciarse en la investigación científica es un proceso complicado. Revista Experiencia en Medicina del Hospital Regional Lambayeque, 4(1), 3335, e-ISSN: 2412-4214. Recuperado de:

http://rem.hrlamb.gob.pe/index.php/REM/article/view/157

Ishiyama-Cervantes, R. (2017a,b,c). Investigación científica tarea agradable. Revista Experiencia en Medicina del Hospital Regional Lambayeque, 3(3), 115-116, e-ISSN: 2412-4214. Recuperado de:

http://rem.hrlamb.gob.pe/index.php/REM/article/view/118

Purizaca-Rosillo, N., Cardoza-Jiménez, K., \& Herrera-Añazco, P. (2016a,b).

Producción científica en una universidad pública peruana beneficiaria del canon. Anales de la Facultad de Medicina, 77(1), 7374, e-ISSN: 1609-9419. Recuperado de:

https://doi.org/10.15381/anales.v77i1.11561

Reynosa, E., Serrano, E., Ortega-Parra, A., Navarro, O., Cruz-Montero, J., \& Salazar, E. (2020). Estrategias didácticas para investigación científica: relevancia en la formación de investigadores. Revista Universidad y Sociedad, 12(1), 259-266, e-ISSN: 2218-3620. Recuperado de:

http://scielo.sld.cu/scielo.php?script=sci arttext\&pid=S2218$\underline{36202020000100259}$ 
Rodríguez, L. (2013). Docencia e investigación: hacia el desarrollo de una cultura de investigación formativa. Investigación Educativa, 17(31), 65-76, e-ISSN: 1728-5852. Recuperado de:

https://revistasinvestigacion.unmsm.edu.pe/index.php/educa/article/vie $\underline{w / 7765}$

Rojas, M. (2015). Tipos de Investigación científica: Una simplificación de la complicada incoherente: nomenclatura y clasificación. REDVET: Revista Electrónica de Veterinaria, 16(1), 1-14, e-ISSN: 1695-7504. Recuperado de:

https://www.redalyc.org/articulo.oa?id=63638739004

Sánchez, H. (2016). Desafíos para la Investigación en la Universidad Peruana. Lima, Perú: Universidad Ricardo Palma. Recuperado de: http://repositorio.urp.edu.pe/handle/urp/677

Stefos, E. (2019). Resultados de investigación de países de América del Sur publicados en revistas indexadas de nivel mundial: Una comparación a nivel internacional. Revista Scientific, 4(12), 9-26, eISSN: 2542-2987. Recuperado de:

https://doi.org/10.29394/Scientific.issn.2542-2987.2019.4.12.0.9-26

SUNEDU (2018). Informe Bienal sobre la Realidad Universitaria Peruana.

1a. edición. Lima, Perú: Superintendencia Nacional de Educación Superior Universitaria.

SUNEDU (2020). II Informe Bienal sobre la Realidad Universitaria en el Perú. ISBN: 978-612-47125-1-7. Lima, Perú: Superintendencia Nacional de Educación Superior Universitaria.

Valles, L. (2015). Nueva Ley Universitaria. Revista de la Sociedad Química del Perú, 81(2), 79-80, e-ISSN: 1810-634X. Recuperado de: http://www.scielo.org.pe/scielo.php?script=sci arttext\&pid=S1810$\underline{634 X 2015000200001}$

Yace, J., Guerrero, J., \& Delgado, J. (2017). Nivel de actitud hacia la 
investigación científica y la estadística en estudiantes de obstetricia pregrado, Universidad Norbert Wiener, agosto 2017. En VIII Simposio Jornada de Investigación. (págs. 37-39). Lima, Perú: Universidad Privada Norbert Wiener. 


\section{Ensayo Original / Original Essay}

José Jesús Guerrero Rojas

e-mail: jguerrero28@ucvvirtual.edu.pe

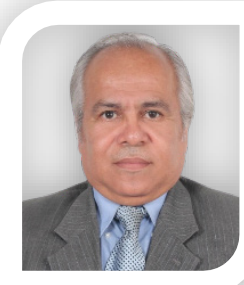

Nacido en la ciudad de Lima, Perú, el 28 de junio del año 1961. Biólogo con mención en Microbiología y Parasitología, egresado de la Facultad de Ciencias Biológicas de la Universidad Nacional Mayor de San Marcos (UNMSM); es Maestro en Docencia Universitaria por la Universidad César Vallejo (UCV); laboro como Docente Universitario en la Universidad Privada Norbert Wiener (UWiener) en EAP de Obstetricia, desde el año 2016 hasta la actualidad; he sido reconocido como Investigador por la EAP Obstetricia; también he sido Docente Tutor en la Universidad César Vallejo, Lima Norte (2011-2014).

El contenido de este manuscrito se difunde bajo una Licencia de Creative Commons ReconocimientoNoComercial-Compartirlgual 4.0 Internacional 